\title{
YAP1/MMP7/CXCL16 axis affects efficacy of neoadjuvant chemotherapy via tumor environment immunosuppression in triple-negative breast cancer
}

\author{
Jia-Qi Yuan^, Ke-Jing Zhang, Shou-Man Wang, Lei Guo \\ Clinical Research Center for Breast Cancer Control and Prevention in Hunan Province, Department of General Surgery, Xiangya Hospital, Central \\ South University, Changsha, China \\ Contributions: (I) Conception and design: JQ Yuan, KJ Zhang, SM Wang, L Guo; (II) Administrative support: SM Wang, L Guo; (III) Provision of \\ study materials or patients: SM Wang, L Guo; (IV) Collection and assembly of data: JQ Yuan, KJ Zhang; (V) Data analysis and interpretation: JQ \\ Yuan; (VI) Manuscript writing: All authors; (VII) Final approval of manuscript: All authors. \\ Correspondence to: Lei Guo. No.87, Xiangya Road, Kaifu District, Changsha 410008, China. Email: kehanshiming@163.com.
}

Background: To evaluate the association of potential YAP1/MMP7/CXCL16 axis and tumor infiltrating lymphocytes (TILs) related chemo-response in triple-negative breast cancer (TNBC) patients.

Methods: We estimated the messenger RNA (mRNA) expression levels of Yes-associated protein 1 (YAP1), MMP7, and CXCL16 in paired TNBC tumor/para-tumor tissues by quantitative real-time reverse transcription polymerase chain reaction (qRT-PCR), and performed statistical analysis according to neoadjuvant chemotherapy (NAC) response. Based on The Cancer Genome Atlas (TCGA) data, we noticed outstanding expression of MMP7/CXCL16 in TNBC cases, as well as associations between MMP7/CXCL16 and HIPPO-YAP1-relevant kinases. We also performed gene set enrichment analysis (GSEA) between MMP7/CXCL16 and YAP1-associated pathways. Western blotting assay was employed to evaluate YAP1/MMP7/CXCL16 expression in vitro and their modulation sequence. Logistic model stepwise regression analysis was used to assess YAP1, MMP7, CXCL16, and TILs as therapeutic predictors. Residual cancer burden (RCB) score was calculated and statistically analyzed according to intensity of these variables, and receiver operating characteristic (ROC) curve also showed their predictive value in NAC response. Recruitment efficacy for CD4+/CD8+ TIL cells (TCGA data) as well as quantified TIL cells density were both explored according to YAP1, MMP7, and CXCL16 expression level.

Results: Up-regulation of YAP1/MMP7 and down-regulation of CXCL16 were both significant in TNBC cases with poor NAC response. Inhibition of YAP1 induced down-regulation of MMP7 and up-regulation of CXCL16, whereas inhibition of MMP7 also induced up-regulation of CXCL16. It was also shown that MMP7/ CXCL16 was enriched in the YAP1-related pathway. Activation of the YAP1/MMP7/CXCL16 axis obviously affected RCB of TNBC cases. The ROC curve also supported the predictive value of YAP1/MMP7/CXCL16 axis and TILs density in NAC response prospect. The density of TILs, meanwhile, demonstrated a strong link with the YAP1/MMP7/CXCL16 axis. Over expression of YAP1/MMP7 significantly suppressed recruitment of CD4+/ CD8+ TILs, while CXCL16 over expression had a beneficial impact on anti-tumor immune.

Conclusions: Over expression of causes up-regulation of MMP7 and down-regulation of CXCL16, which suppressed CD4+/CD8+ TILs recruitment and indirectly affected NAC response of TNBC patients.

Keywords: Tumor-infiltrating lymphocyte (TILs); triple negative breast cancer (TNBC); neoadjuvant chemotherapy (NAC); Yes-associated protein 1 (YAP1); matrix metalloproteinases (MMPs)

Submitted Aug 05, 2021. Accepted for publication Sep 17, 2021.

doi: $10.21037 / g s-21-612$

View this article at: http://dx.doi.org/10.21037/gs-21-612

^ ORCID: 0000-0002-4369-8096. 


\section{Introduction}

Activation of the immune pathway and immune microenvironment could improve neoadjuvant chemotherapy (NAC) efficacy and tumor remission (1,2). Immunogenic cell death has been shown to result in antigen crosspresentation, activation of dendritic cells (DCs), and induction of tumor-specific cytotoxic T cells (3). Tumorinfiltrating lymphocytes (TILs) have been shown to be associated with outcomes of breast cancer (4), and are considered a reliable marker of chemotherapy efficacy in triple negative breast cancer (TNBC) (5). Randomized trials have confirmed that TILs are usually sufficient in highly proliferative tumors and that their presence has been associated with excellent pathologic response $(6,7)$.

As micro-environment related factors, increasing studies have reported associations between matrix metalloproteinases (MMPs) and anti-tumor immune functions, such as processing CCL/CXCL chemotaxis, modulating antitumor immune responses, and inducing tumor immune escape. Based on our previous work, Yesassociated protein 1 (YAP1) functions as crucial predictor of NAC efficacy. Reports have also demonstrated a strong link between YAP1 and chemotherapy sensitivity. However, few studies have focused on the relationship between YAP1 and CXCL chemotaxis, as well as their interaction in the tumor immune micro-environment. Considering the over expression of MMP7 and CXCL16 in The Cancer Genome Atlas (TCGA)-TNBC data, we evaluated the potential association of the YAP1/MMP7/CXCL16 axis and TILs-related chemo-response in TNBC patients. We present the following article in accordance with the MDAR reporting checklist (available at https://dx.doi. org/10.21037/gs-21-612).

\section{Methods}

\section{Patients and samples}

A total of 433 consecutive cases of TNBC, diagnosed and treated between June 2017 and January 2020, were retrieved from the database of Central-South University Xiangya Hospital Breast Cancer Center. Patients with inflammatory breast cancer, distant metastasis, or bilateral breast tumors were not included. All patients were diagnosed via core needle biopsy, treated with anthacycline and taxane based NAC, and underwent surgery, namely, breast-conserving or mastectomy + sentinel lymph node biopsy or modified radical mastectomy, with or without radiation therapy.
Disease characteristics were collected from hospital medical records, including age at diagnosis, clinical tumor, node, metastasis (cTNM) stage, histological grade, Ki67 indication, lymph-vascular space invasion (LVSI), systemic chemotherapy regimen, NAC response, and lymph nodes metastases (Table 1). The study was conducted in accordance with the Declaration of Helsinki (as revised in 2013) and was approved by the Clinic Institutional Review Board of Xiangya Hospital. All participants gave our group permission to use their tissue specimens by signing informed consent.

All specimens extracted from the tumor by core biopsy were fixed with $10 \%$ neutral phosphate-buffered formalin and paraffin-embedded. Slices ( $4 \mu \mathrm{m}$ thick) of tumor blocks were stained with hematoxylin and eosin (H\&E). According to the immunohistochemistry (IHC) staining results, hormone receptor negative was defined as estrogen receptor (ER) $<1 \%$ and progesterone receptor $(\mathrm{PR})<1 \%$. Human epidermal growth factor receptor 2 (HER-2) negative was defined as IHC staining $1+$ or 0 . The TNBC was defined as ER, PR, and HER2 negative. We employed a relevant antibody and performed western blotting to evaluate YAP1 (Affinity Cat\# BF0368, RRID: AB_2833985), MMP7 (Affinity Biosciences, Cincinnati, OH, USA; Cat\# AF0218, RRID: AB_2833348), and CXCL16 (Affinity, Cat\# DF13312, RRID: AB_2846331) expression in MDA-MB-231 (American Type Culture Collection (ATCC), Manassas, VA, USA; Cat\# CRMHTB-26, RRID:CVCL_0062), and HCC70 (ATCC, Cat\# CRL-2315, RRID: CVCL_1270) cell lines, respectively. We also compared messenger RNA (mRNA) expression levels via quantitative real-time reverse transcription polymerase chain reaction (qRT-PCR) in paired tumor/para-tumor tissues, and performed statistical analysis in response (R) and no response (NR) cases, respectively.

\section{Pathologic assessment of TILs}

All cases were diagnosed as invasive breast cancer by experienced breast pathologists from our hospital. Slides with a standard thickness of $4-5 \mu \mathrm{m}$ were observed via a microscope magnification of $200 x-400 \times$ (ocular 10x, with an objective of $20 x-40 x$ ) field. Histopathologic evaluation of stromal TILs (sTIL) were evaluated according to the criteria recommended by the International TILs Working Group 2014 (8). Interpersonal discussions were permitted when the scoring was controversial. The TILs were assessed within the borders of invasive tumors, and stromal TILs 
Table 1 Demographic information of the patients in this study

\begin{tabular}{|c|c|c|}
\hline Characteristic & No. $(n=433)$ & $\%$ \\
\hline \multicolumn{3}{|l|}{ Age (years) } \\
\hline Median [range] & 43 [19-81] & - \\
\hline$\leq 50$ & 295 & 68.13 \\
\hline$>50$ & 138 & 31.87 \\
\hline \multicolumn{3}{|l|}{ cTNM stage } \\
\hline II & 40 & 9.24 \\
\hline III a & 291 & 67.21 \\
\hline III b & 102 & 23.55 \\
\hline \multicolumn{3}{|l|}{ Histological grade } \\
\hline 1 & 119 & 27.48 \\
\hline 2 & 239 & 55.20 \\
\hline 3 & 75 & 17.32 \\
\hline \multicolumn{3}{|l|}{ Ki67 score (\%) } \\
\hline$>14 \%$ & 213 & 49.19 \\
\hline$\leq 14 \%$ & 220 & 50.81 \\
\hline \multicolumn{3}{|l|}{ LVSI status } \\
\hline+ & 252 & 58.20 \\
\hline- & 181 & 41.80 \\
\hline \multicolumn{3}{|l|}{ Lymph nodes after NAC } \\
\hline$>3$ & 106 & 24.48 \\
\hline$\leq 3$ & 327 & 75.52 \\
\hline \multicolumn{3}{|l|}{ Local therapy } \\
\hline Mastectomy + ALND + RT & 47 & 10.85 \\
\hline Mastectomy + ALND & 186 & 42.96 \\
\hline Mastectomy + SLNB + RT & 10 & 2.31 \\
\hline Mastectomy + SLNB & 78 & 18.01 \\
\hline$B C S+A L N D+R T$ & 28 & 6.47 \\
\hline$B C S+S L N B+R T$ & 84 & 18.40 \\
\hline \multicolumn{3}{|l|}{ Pathological response } \\
\hline $\mathrm{pCR}$ & 173 & 39.95 \\
\hline Non-pCR & 260 & 60.05 \\
\hline
\end{tabular}

cTNM, clinical tumor, node, metastasis; LVSI, lymph-vascular space invasion; NAC, neoadjuvant chemotherapy; ALND, axillary lymph node dissection; SLNB, sentinel lymph node biopsy; BCS, breast conserving surgery; RT, radiation therapy; $\mathrm{pCR}$, pathological complete response. were defined as the area occupied by TILs over the total stromal area; TILs beyond the tumor border or around ductal carcinoma in situ (DCIS) were excluded. Areas with crush artifacts, necrosis, and regressive hyalinization were not included. The results were scored as intervals: low scoring (sTIL $=0$ ) was defined as $0-10 \%$; median scoring (sTIL $=1$ ) was defined as $11-20 \%$, and high scoring (sTIL $=2$ ) was defined as $>20 \%$. Antibodies including CD3, CD4, CD8 and FOXP3 were used in the study of TIL subset.

\section{Evaluation of therapeutic response}

We identified pathological complete response (pCR) as no pathological evidence of residual invasive lesions in the breast or axillary lymph nodes. We regarded residual DCIS as pCR in this study. We measured residual tumor size via ultrasound every week, and sought confirmation by magnetic resonance imaging (MRI) at the beginning of every new NAC cycle. As an independently and strongly prognostic indicator for distinct breast cancer phenotypes, we determined residual cancer burden (RCB) continuous index by factors including the dimensions of the primary tumor bed area, percentage of invasive lesions versus lesions in situ, number of involved lymph nodes (LN), and size of the largest metastasis. We classified RCB as 0 (no residual tumor), I (minimal burden), II (moderate burden), and III (extensive burden). The calculation formula and detailed description about RCB was located at a free and dedicated website: http://www.meaderson.org/breastcancer_RCB.

\section{Bioinformatics analysis}

We assessed differential expression of MMP7 and CXCL16 in TCGA database, including tumor/para-tumor tissues of TNBC cases as well as distinct molecular subgroups. We performed gene set enrichment analysis (GSEA) to evaluate association between MMP7/CXCL16 expression and HIPPO-YAP1 signal pathway. We also estimated association between MMP7/CXCL16 and HIPPO-YAP1 relevant kinases in mRNA expression level. We explored the TIL-recruitment effect of YAP1/MMP7/CXCL16 in TNBC cases from TCGA database.

\section{Statistical analyses}

We used logistic model stepwise regression analysis to 
evaluate therapeutic effect of variables, expressing the results as hazard ratios (HRs) and 95\% confidence intervals (CIs). Receiver operating characteristic (ROC) curves were performed respectively to quantify associations between relevant variables and therapeutic response of NAC. All statistical tests were 2 -sided and $\mathrm{P}$ values less than 0.05 were considered statistically significant. Statistical analysis was carried out using SPSS version 25.0 for Windows (SPSS Inc., Chicago, IL, USA). We used GraphPad Prism 8.0 (GraphPad Software Inc., San Diego, CA, USA) to create figures.

\section{Results}

We compared YAP1 expression of paired tumor/para-tumor tissues in R/NR TNBC cases. As shown in Figure 1A, upregulation of YAP1 expression was significant in poor response cases, which suggested an outstanding association between YAP1 expression and NAC response in TNBC patients. We also compared TILs density in R/NR cases as well as YAP1 poor/well expression subgroups. As shown in Figure $1 \mathrm{~B}$, TILs tended to concentrate in excellent remission cases and YAP1 insufficient cases, suggesting some kind of reverse trend between YAP1 intensity and TILs score.

According to the analysis results of TCGA database, we observed notable differential expression of MMP7 and CXCL16 in paired tumor/para-tumor TNBC tissues. As shown in Figure 2A,2B, tumor-expression MMP7 and CXCL16 were more remarkable than in para-tumor tissues, and TNBC cases revealed obvious differential expression in comparison with other molecular types. Furthermore, we estimated MMP7 and CXCL16 expression in NR cases. As shown in Figure 2C,2D, up-regulation of MMP7 and downregulation of CXCL16 were both significant in tumor tissues from poor response patients, which meant potential reverse therapeutic effect of MMP7 and CXCL16.

Based on the above findings, we suspected a relationship between MMP7/CXCL16 and YAP1 expression. Thus, we performed GSEA between MMP7/CXCL16 and YAP1-associated pathway. As shown in Figure 3, MMP7 and CXCL16 expression showed a positive correlation with HIPPO-YAP1 pathways. We also observed a close association between MMP7/CXCL16 expression and HIPPO-YAP1 relevant kinases (such as MST1 and LATS2). These results implied that YAP1 intensity regulated MMP7/ CXCL16 expression, thereby affecting the NAC response in TNBC patients.
Considering the potential existence of YAP1-MMP7CXCL16 axis in gene enrichment and mRNA expression level, we assessed the probable association in protein expression level via MDA-MB-231 and HCC70 cell lines, respectively. As shown in Figure 4, verteporfin (YAP1 inhibitor) suppressed expression of MMP7 and improved expression of CXCL16, whereas GM6001 (MMPs inhibitor) only improved the expression of CXCL16. These results identified a similar regulatory relationship of protein expression in accordance with gene enrichment and mRNA expression, thereby demonstrating the modulation sequence in YAP1-MMP7-CXCL16 axis.

Furthermore, we estimated association between the above variables and RCB score via logistic model stepwise regression analysis. As shown in Figure 5A, prolific density of TILs and CXCL16 expression could improve NAC response, while over expression of YAP1 and MMP7 suppressed tumor remission. These 4 variables significantly affected TNBC patients' RCB after NAC (Figure 5B), owning fantastic sensitivity and specificity (Figure 5C).

Forasmuch as the notable association of TILs density and NAC response, we investigated the immune cells recruitment effect of the YAP1-MMP7-CXCL16 axis. According to the analysis results of TCGA database (Figure $6 \mathrm{~A}$ ), we observed a remarkable positive association between CXCL16 expression and CD4+/CD8+ t cells infiltration. In comparison, the negative correlation between YAP1/MMP7 expression and CD4+/CD8+ t cells infiltration was similarly outstanding. We also quantified TILs density according to YAP1/MMP7/CXCL16 intensity. According to our microscopic observation in this study, sufficient CD4+/CD8+TILs counts closely coincided with the over expression of CXCL16 as well as the inferiority of YAP1/MMP7 (Figure 6B).

In order to explore the relationship between TIL subgroups and therapeutic response, we estimated the cell types and expression characteristics in the immune microenvironment of these TNBC cases. As shown in Figure 7A, ratio of $\mathrm{CD} 3+$ and CD8+ TILs was observably higher in primary lesions of well-remission cases. Correspondingly, we also noticed outstanding insufficiency of CD3+ and CD8+ TILs in the residual lesion (Figure $7 B$ ). These results suggested that CD3+ and CD8+ TILs significantly contributed to the pathological remission.

As the primary trigger of the whole signal pathway, we further estimated the prognostic significance of YAP1 over-expression. Based on 4934 cases from GSE20685, GSE25066, GSE41998, GSE2034 3494 and other 43 data 
A
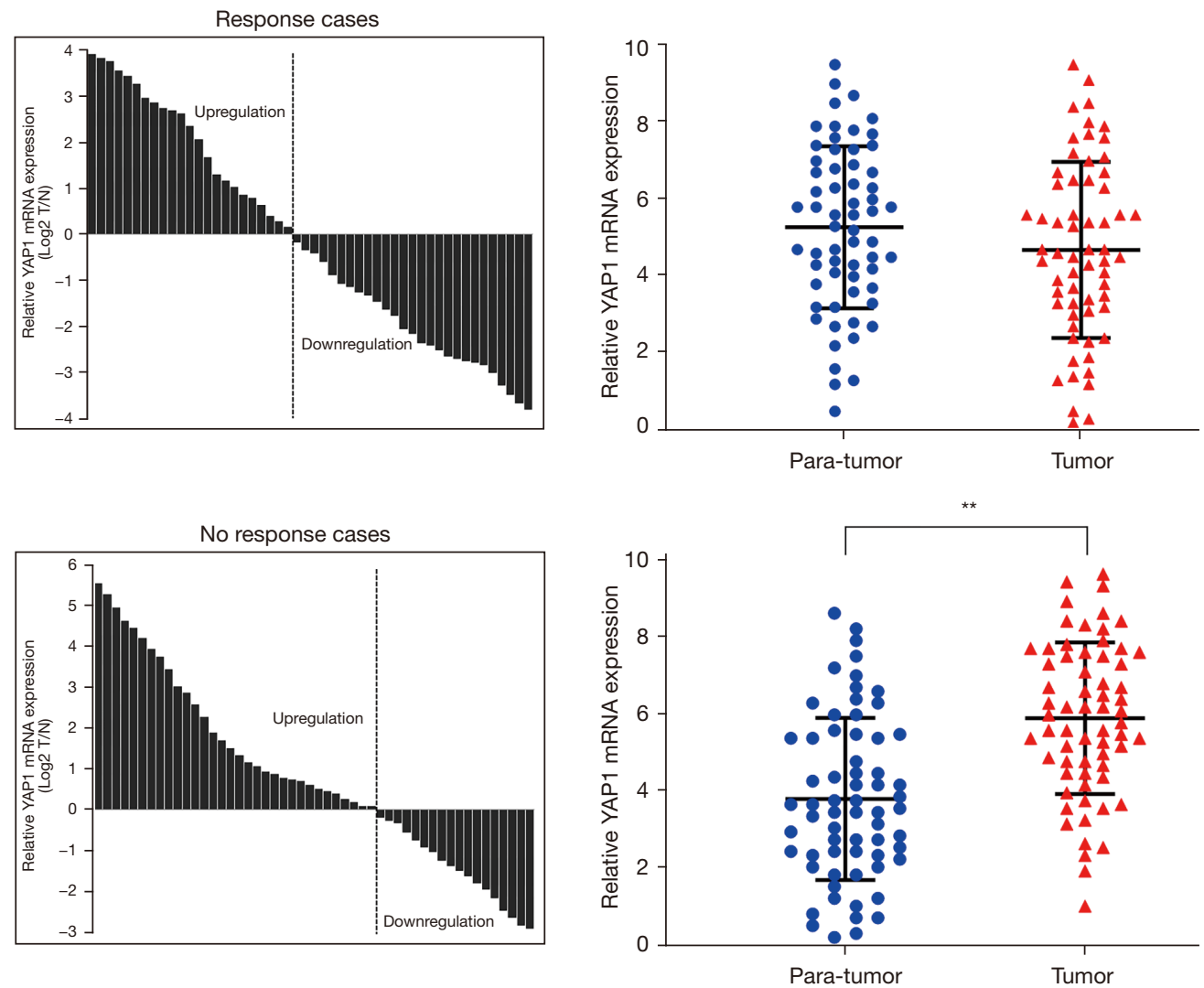

B

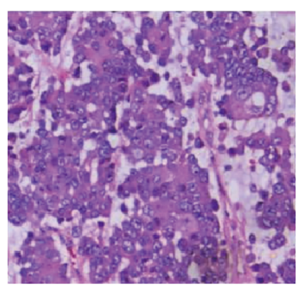

YAP1 (+)

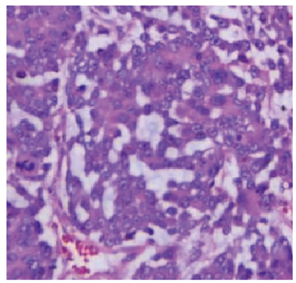

NR

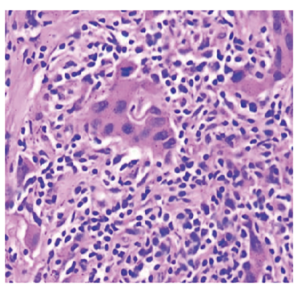

YAP1 (-)

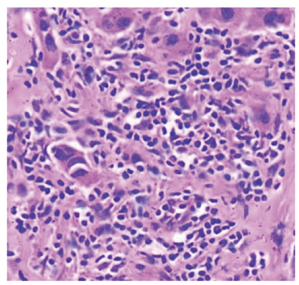

$\mathrm{R}$
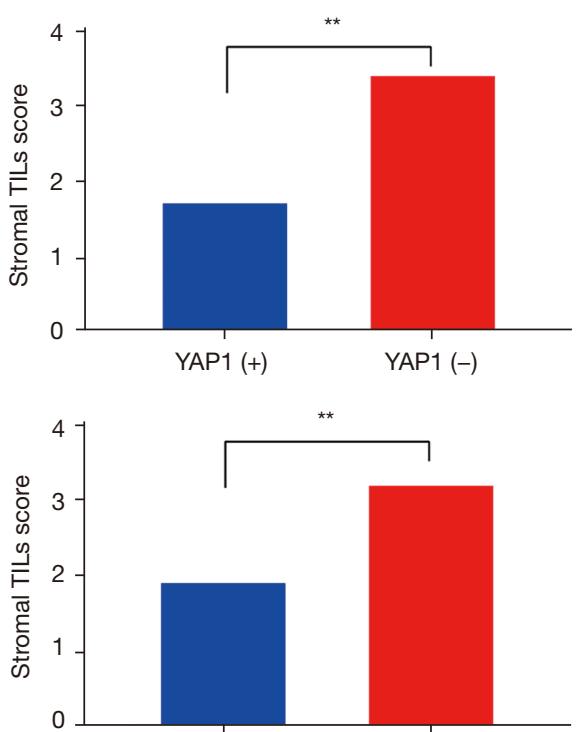

NR

R

Figure 1 Relationship between YAP1 intensity and therapeutic response. (A) Expression levels of YAP1 mRNA in paired TNBC and adjacent normal tissues were evaluated by qRT-PCR. Statistical analysis was performed in NAC response group and no response group, respectively (**, P $<0.01)$. (B) Representative H\&E-stained images of TILs density $(\times 200)$ was compared and statistical analyzed according to YAP1 intensity and therapeutic response, respectively (** $\mathrm{P}<0.01)$. R, response; NR, no response; mRNA, messenger RNA; TNBC, triplenegative breast cancer; qRT-PCR, quantitative real-time reverse transcription polymerase chain reaction. 

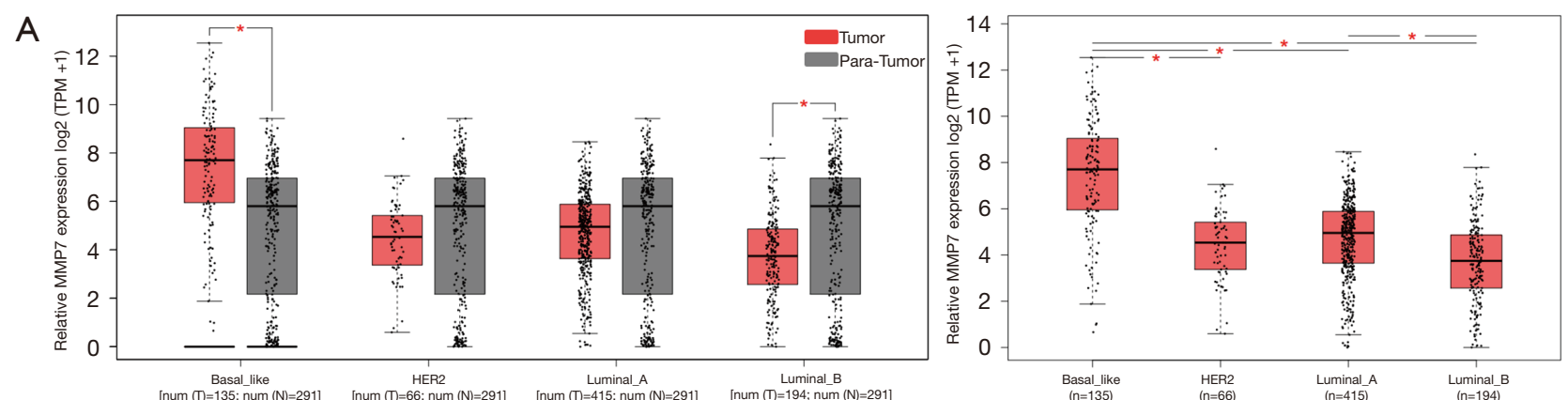

B
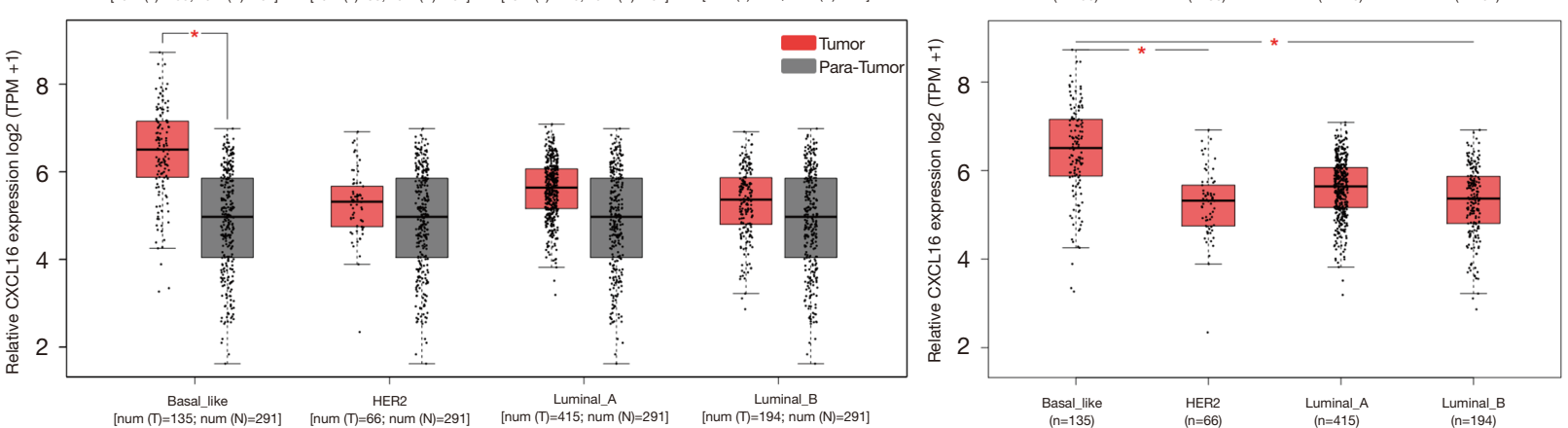

C
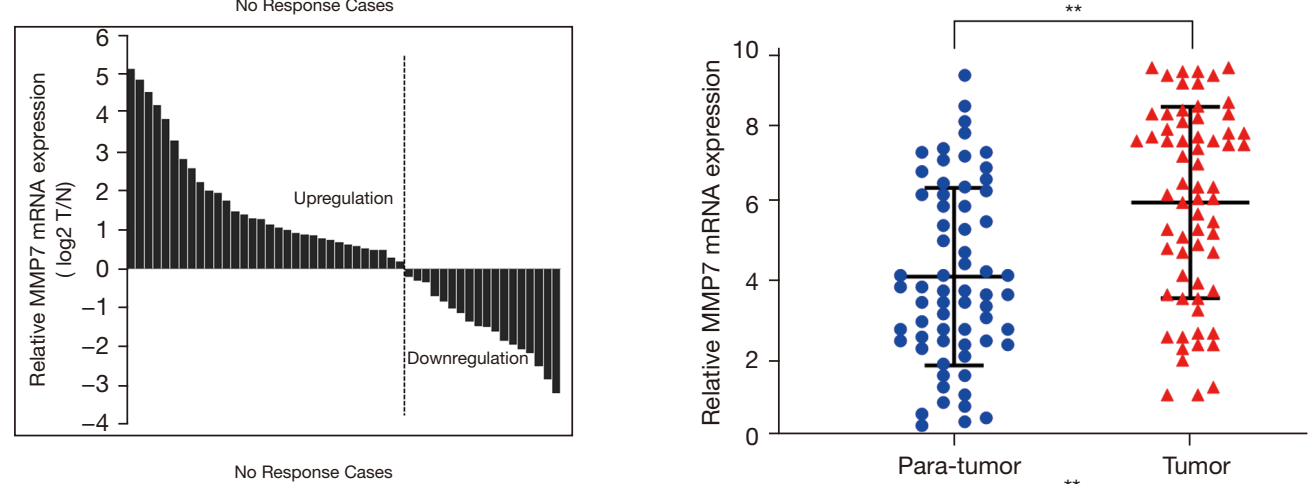

D
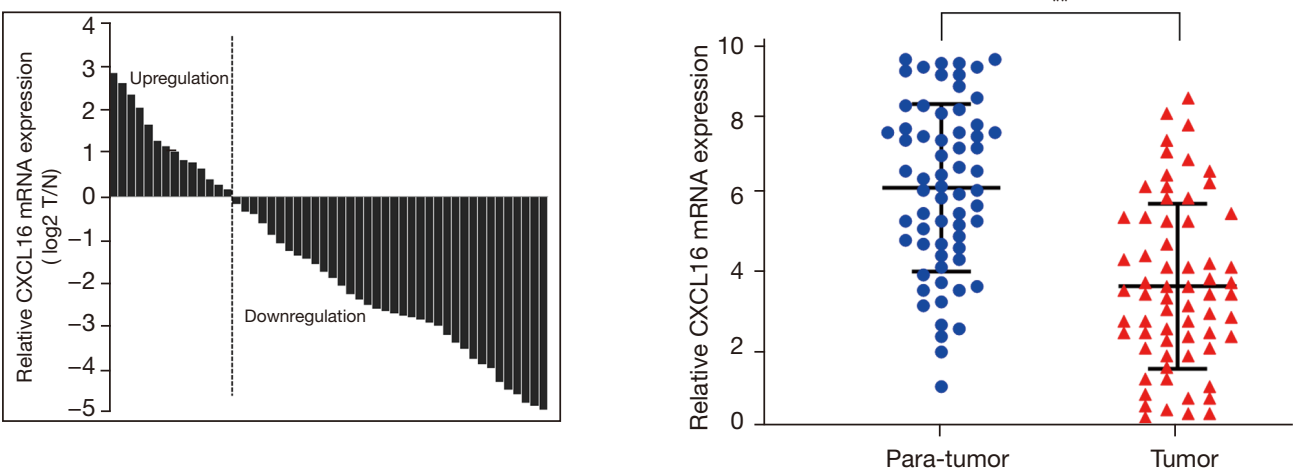

Figure 2 Relationship between MMP7/CXCL16 expression and therapeutic response of TNBC. (A) Expression levels of MMP7 mRNA in TCGA breast cancer database were evaluated according to molecular types (TCGA) $\left({ }^{*}, \mathrm{P}<0.05\right)$. (B) Expression levels of CXCL16 mRNA in TCGA breast cancer database were evaluated according to molecular types $\left({ }^{*}, \mathrm{P}<0.05\right)$. (C) Expression levels of MMP7 mRNA in paired TNBC and adjacent normal tissues were evaluated by qRT-PCR. Statistical analysis was performed in no response cases (**, P<0.01). (D) Expression levels of CXCL16 mRNA in paired TNBC and adjacent normal tissues were evaluated by qRT-PCR. Statistical analysis was performed in no response cases $\left({ }^{* *}, \mathrm{P}<0.01\right)$. mRNA, messenger RNA; TNBC, triple-negative breast cancer; qRT-PCR, quantitative realtime reverse transcription polymerase chain reaction; TCGA, The Cancer Genome Atlas. 

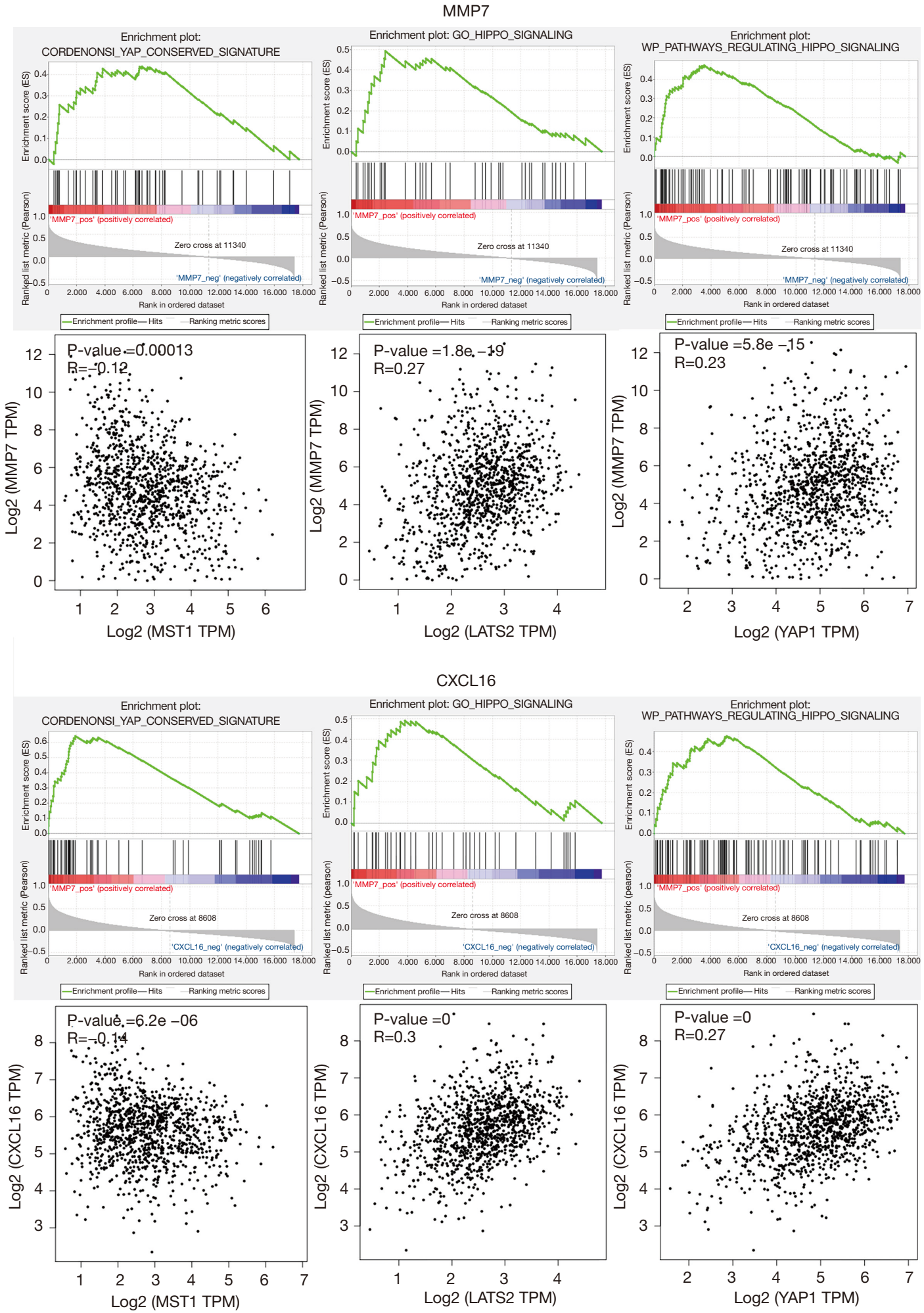

Figure 3 GSEA between MMP7/CXCL16 and YAP1-associated pathway was performed using TCGA data. Associations between MMP7/ CXCL16 expression and HIPPO-YAP1 relevant kinases (such as MST1 and LATS2) were also explored according to TCGA data. GSEA, gene set enrichment analysis; TCGA, The Cancer Genome Atlas. 

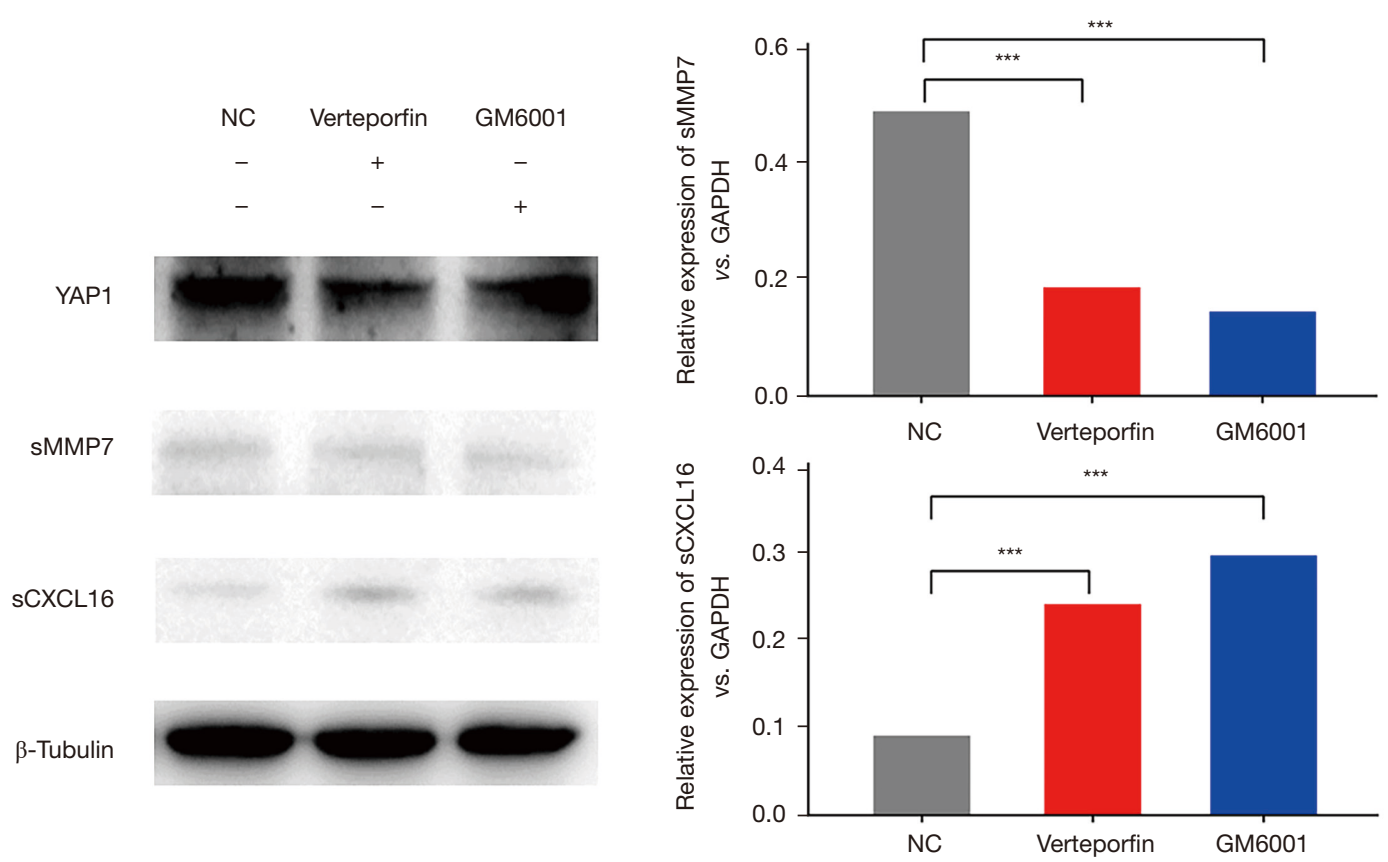

MDA-MB-231
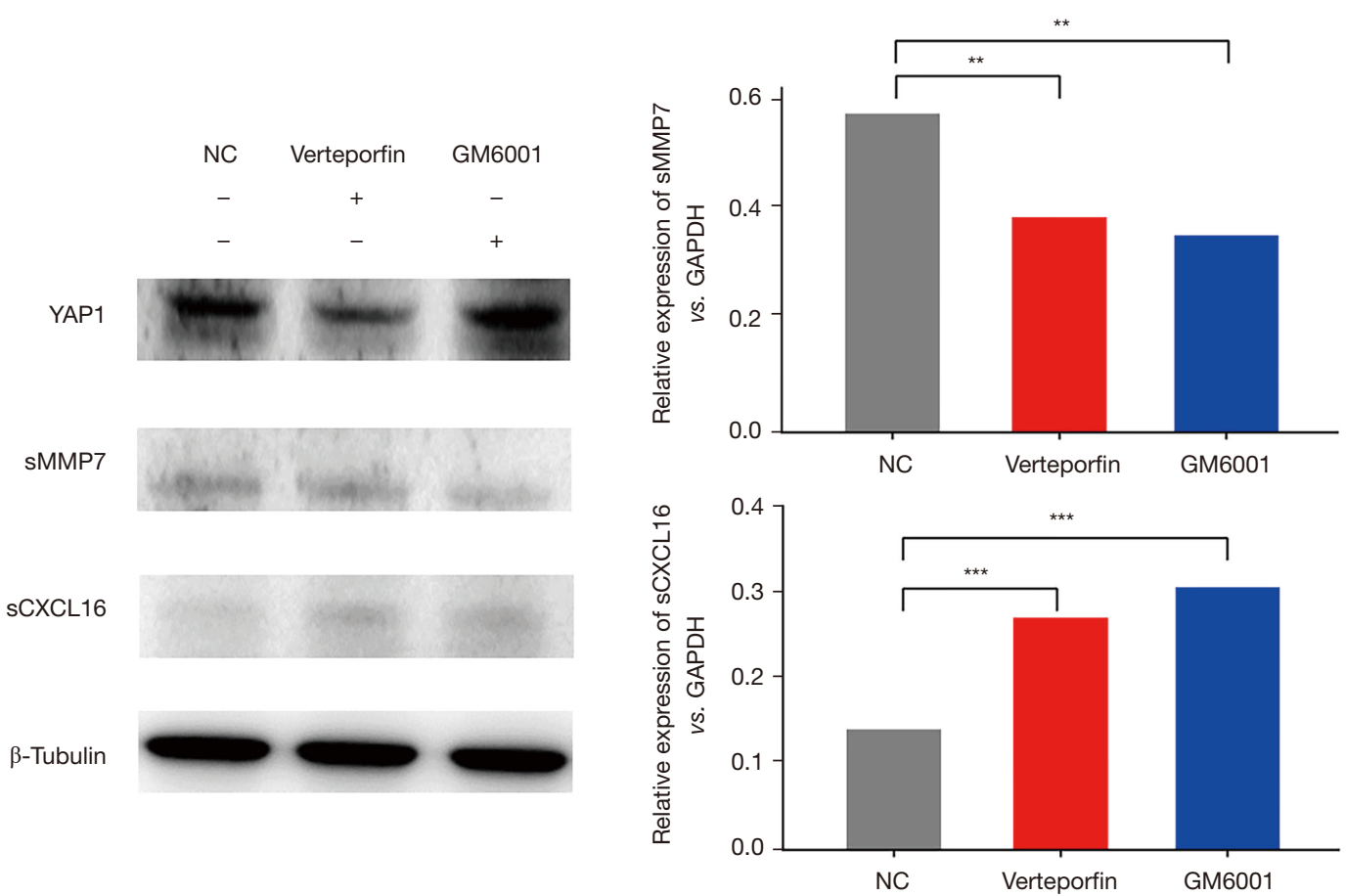

$\mathrm{HCC70}$

Figure 4 Western blotting assay was employed to measure cytoplasmic YAP1 as well as soluble MMP7 and CXCL16 in extracellular matrix in MDA-MB-231 and HCC70 cell lines, respectively. Modulation sequence of protein expression was estimated via YAP1 inhibitor (verteporfin) and MMPs inhibitor (GM6001). Results were statistically analyzed $\left({ }^{* *}, \mathrm{P}<0.01 ;{ }^{* *}, \mathrm{P}<0.001\right)$. 
A

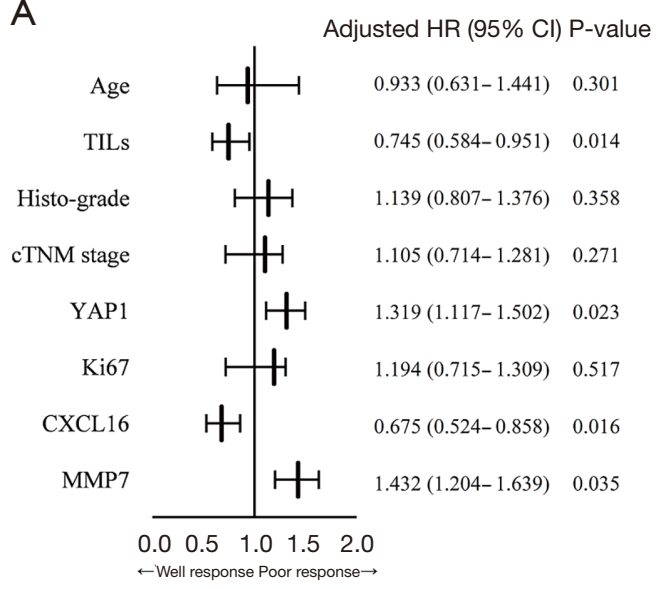

C
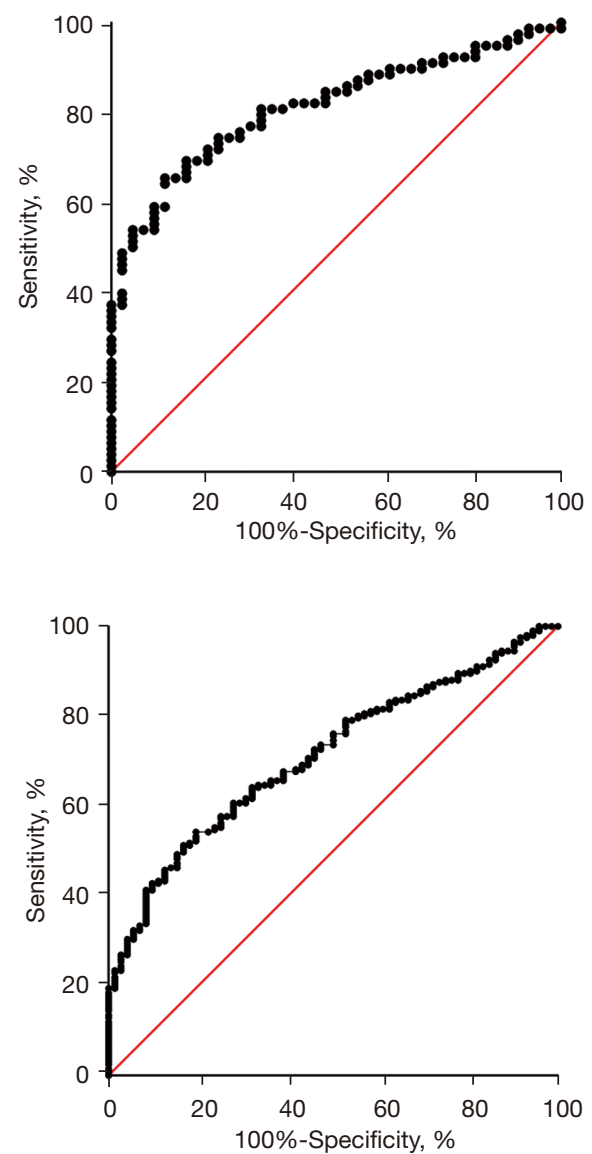

B
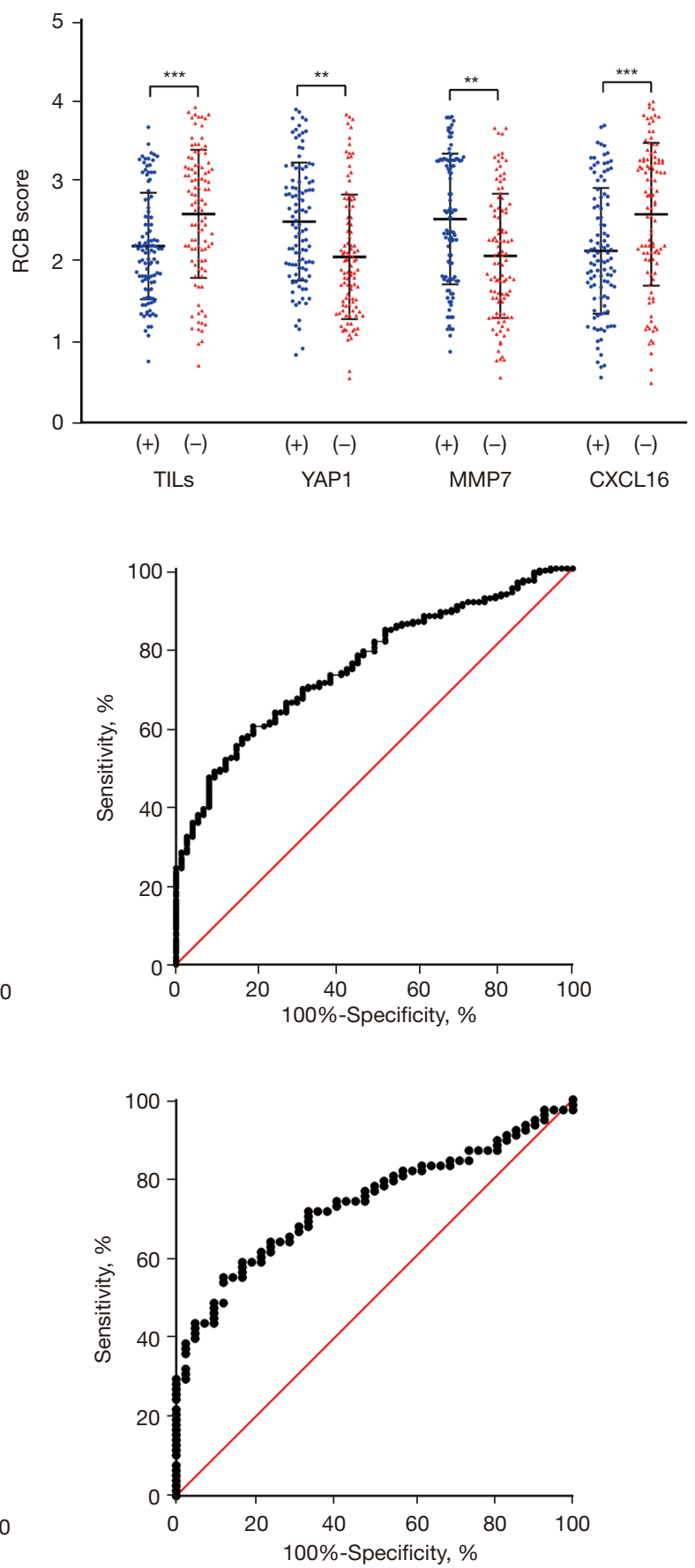

Figure 5 Therapeutic response evaluation based on TILs and YAP1/MMP7/CXCL16 axis. (A) logistic model stepwise regression analysis was employed to investigate variables as therapeutic predictors, which demonstrated the beneficial effects of sufficient TILs/CXCL16 as well as the adverse effects of sufficient YAP1/MMP7 on tumor remission. (B) RCB score was calculated and statistically analyzed according to TILs density and YAP1/MMP7/CXCL16 intensity, respectively (**, $\mathrm{P}<0.01$; ${ }^{* *}, \mathrm{P}<0.001$ ). (C) ROC curve proved the predictive value of YAP1, MMP7, CXCL16, and TILs density in NAC response prospect. TILs, tumor infiltrating lymphocytes; RCB, residual cancer burden; ROC, receiver operating characteristic; NAC, neoadjuvant chemotherapy. 
A
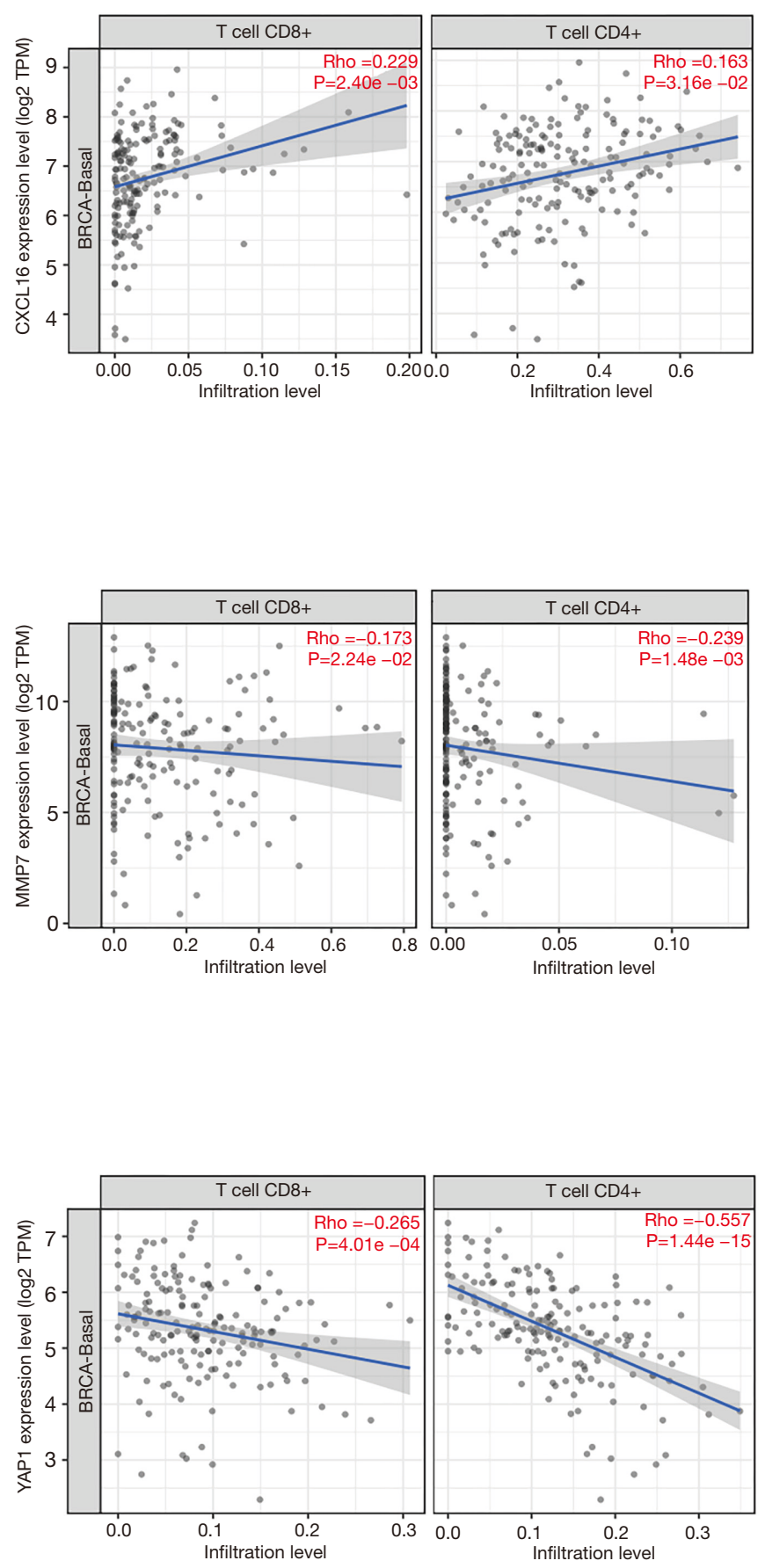
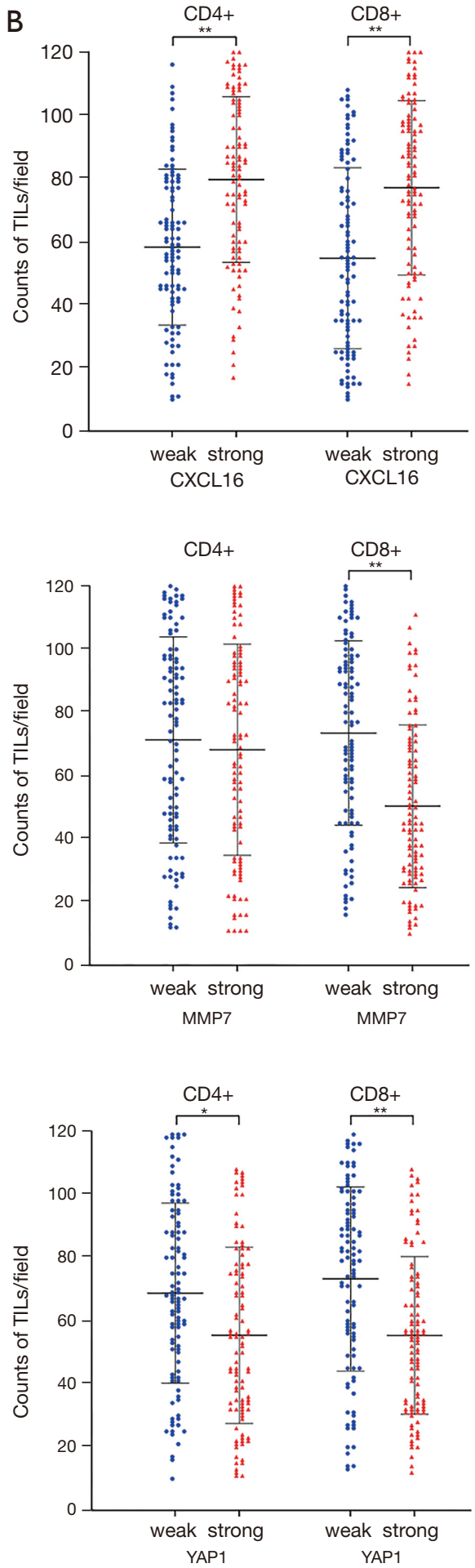

Figure 6 Recruitment effect of YAP1/MMP7/CXCL16 axis for CD4+/CD8+ TIL cells. (A) Recruitment for CD4+/CD8+ TIL cells was explored according to YAP1, MMP7, and CXCL16 expression levels, respectively (TCGA database). (B) Microscopic observation was also performed to quantified CD4+/CD8+ TIL cells density according to YAP1, MMP7, and CXCL16 intensity, respectively $\left(^{*}, \mathrm{P}<0.05\right.$; **, $\mathrm{P}<0.01)$. TCGA, The Cancer Genome Atlas; TIL, tumor infiltrating lymphocyte. 


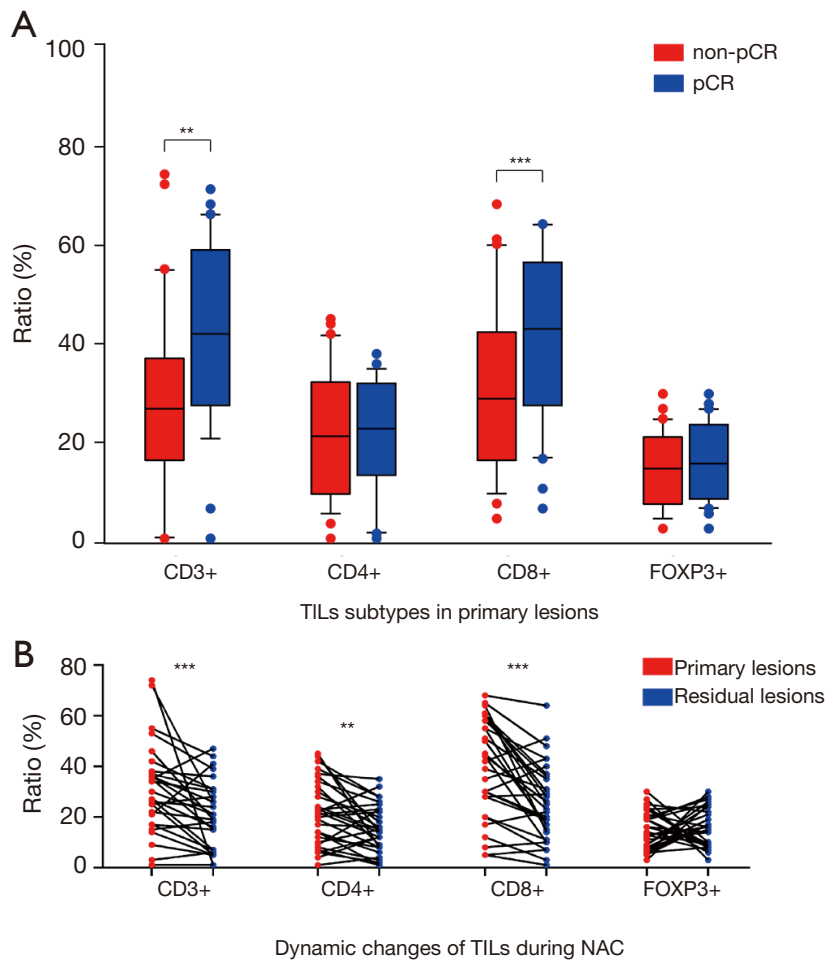

Figure 7 TILs subsets and their dynamic changes in the NAC. (A) Ratio of CD3 + and CD8+ TILs was observably higher in primary lesions of well-remission cases (**, $\mathrm{P}<0.01$; ${ }^{* * *}, \mathrm{P}<0.001$ ). (B) $\mathrm{CD} 3+$ and CD8+ TILs in the residual lesion were significantly decreased after NAC $\left({ }^{* *}, \mathrm{P}<0.01\right.$; $\left.{ }^{* * *}, \mathrm{P}<0.001\right)$. NAC, neoadjuvant chemotherapy; TIL, tumor infiltrating lymphocyte.

sets, we noticed the poor relapse free survival and overall survival of YAP1 sufficient cases (Figure 8A). Similarly, high YAP1 intensity led to unsatisfied outcome in people who underwent radiotherapy (Figure 8B). According to our own clinical practice and follow-up, therapeutic efficacy of radiotherapy was significantly weakened by intensified YAP1 expression (Figure 8C).

To be expected, we observed a 5 -year relapse free survival of $86.2 \%$ in well pathological remission cases and proved residual disease as an independent recurrent risk factor (HR 9.88, 95\% CI: 1.79-23.04), which was consistent with previous studies. Considering the above crucial role of chemotherapeutic response in prognosis of TNBC, we further explored the recurrent risk of cases which suffered residual tumor as well as unsatisfied chemotherapeutic sensitivity, and we compared the clinical and immune characteristics between their primary and residual lesions. Interestingly, the prognostic effect of tumor size and lymph node status was remarkable in both primary and residual lesions (Figure 8D), whereas the prognostic effect of YAP1 and TILs was more outstanding in residual lesions (Figure 8E).

\section{Discussion}

It has become clear that the tumor micro-environment, and, in particular the immune system, plays a crucial role in modulating tumor progression and response to therapy. In fact, some types of chemotherapies have been confirmed to reduce the number of T-regulatory (Treg) cells, which have been shown to be immune suppressive in the tumor, thus allowing a more immune-favorable environment to form and clear a path for effector and memory $\mathrm{T}$ cell responses to destroy cancer cells (9). There has been evidence that suggests that the phenotype and function of the immune infiltrates, in tumors, markedly affect prognosis and patient outcome might be predicted by the characteristics of the anti-cancer specific immune responses (10). Recent insights into the process of how tumors acquire an immunosuppressive environment reinforced the hypothesis that an anti-tumor effector response, such as of the CD8+ $\mathrm{T}$ cell response, took place, but was possibly abrogated prematurely due to a negative feedback response (11). This hypothesis was also supported by studies showing that the degree of TILs was predictive of a better local response to chemotherapy and was prognostic of long-term disease control (12). Furthermore, the absence of TILs was shown to be associated with a poorer disease-free survival and overall survival (13). The TILs were represented mainly by non-activated $\mathrm{T}$ cells, and exposure to chemotherapy induced activation in a significant proportion of cases, even converting a TIL- tumor into a TIL+ tumor (14). These chemotherapy-triggered $\mathrm{T}$ cells were required to prevent a small number of chemotherapy surviving tumor cells from re-establishing a new tumor (15). In this study, TILs tended to concentrate in excellent remission cases. We observed that prolific density of TILs improved NAC response. The ROC curve also revealed the predictive value of TILs density in NAC response prospect. These findings were consistent with the abovementioned reports.

The unique CXC chemokine CXCL16 could function as a chemoattractant as well as a cell adhesion molecule for cells expressing CXCR6 (16), and CXCL16 could possess multiple biological activities both in a transmembrane form and a soluble form (17). Previous studies have shown the antitumor effects of chemokines. The concept was 
A

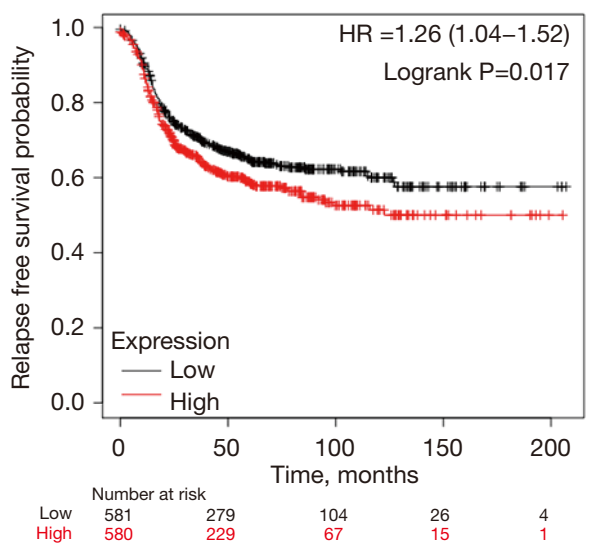

B

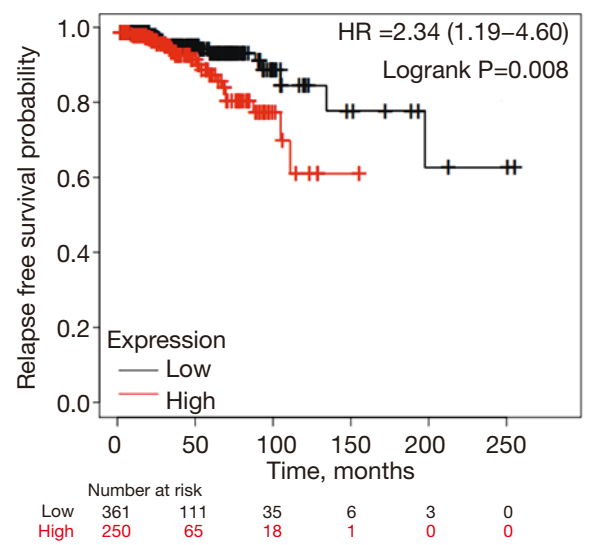

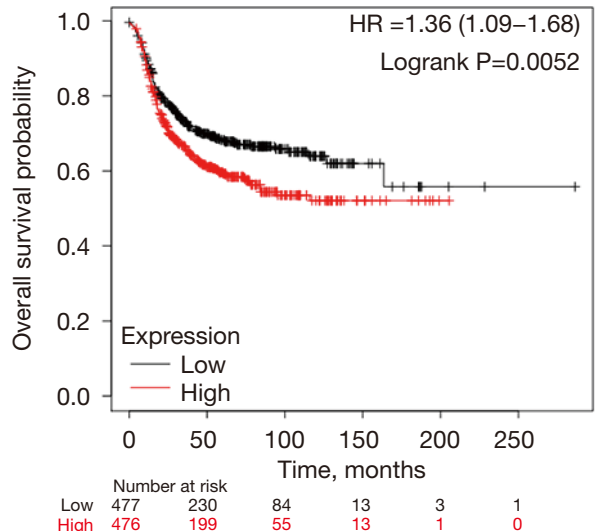

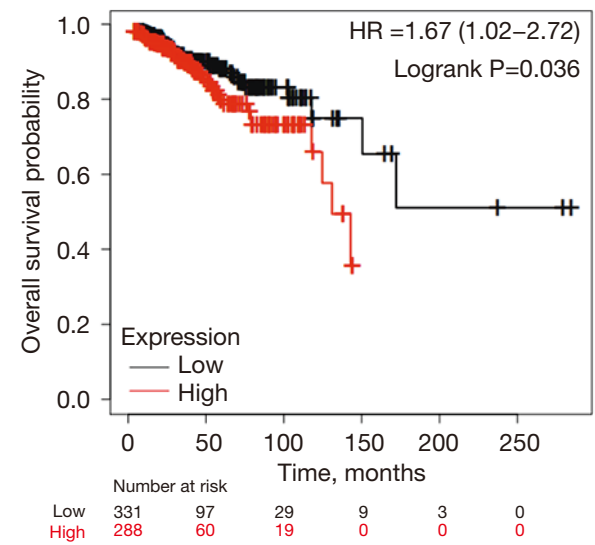

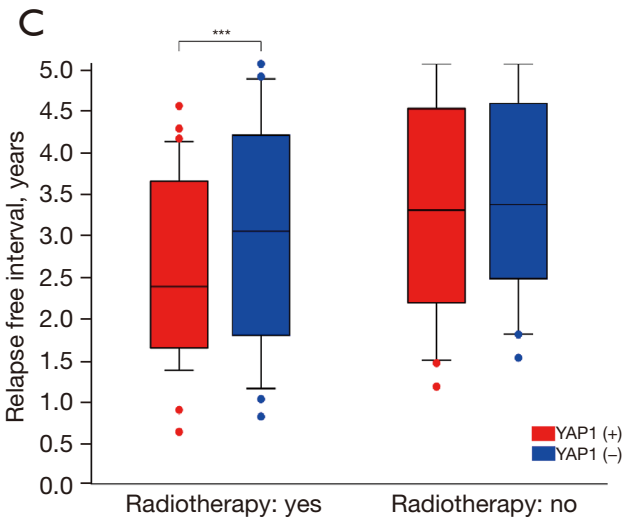

$\mathrm{D}$

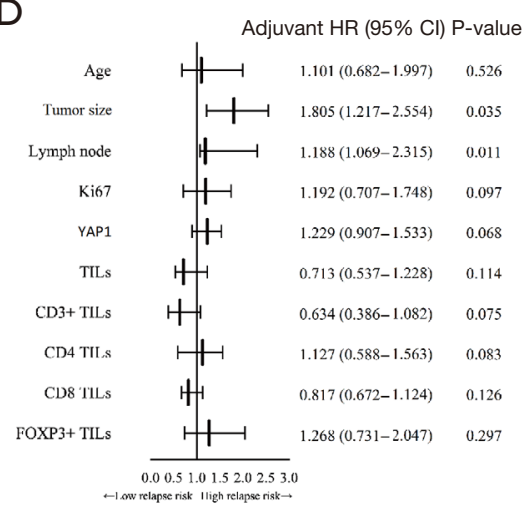

$E$

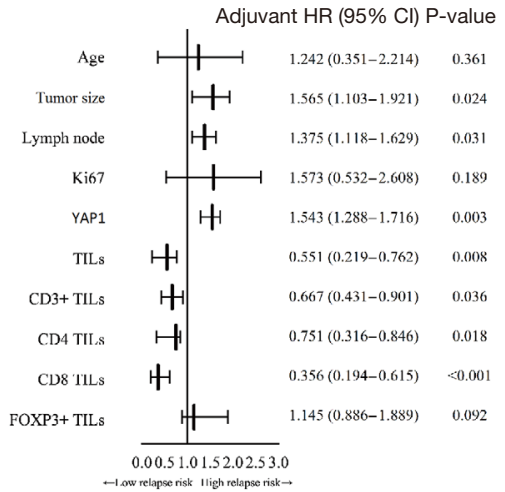

Figure 8 Prognostic effect of clinical and immune factors. (A) YAP1 sufficient cases generally suffered poor relapse free survival and overall survival. (B) High YAP1 intensity caused unsatisfied survival even in patients who underwent radiotherapy. (C) YAP1 sufficient cases also suffered unsatisfied radiotherapy efficacy according to our own clinical practice and follow-up (***, P<0.001). (D) Advanced tumor size and lymph node status were both relapse risk factors in primary and residual lesions. (E) Prognostic effect of YAP1 and TILs intensity was more outstanding in residual tumors. 
that the expression of soluble CXCL16 at tumor tissues could attract immune cells bearing relevant receptor CXCR6 (18), such as CD4+ T cells, CD8+ T cells, and natural killer (NK) cells (19). Cell surface-anchored CXCL16 could also adhere to these CXCR6-expressing cells (20). The above chemo-attractant effect would possibly lead to the induction of antitumor immunity. Outstanding tumor suppressive ability has been tested for CCL3,

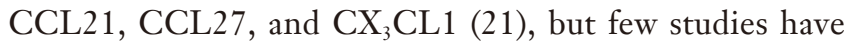
reported whether CXCL16 had similar antitumor activity in breast cancer. It also remained to explore why breast cancer cells up-regulated CXCL16. Some studies suggested that CXCL16 expression at the tumor site might exert a potent immune suppressive effect on cancer growth progression induced by transforming growth factor- $\beta$ (TGF- $\beta$ ), thereby representing a crucial self-check mechanism against tumor progress (22). In this study, we observed significant downregulation of CXCL16 expression in poor remission cases. Interestingly, over expression of CXCL16 was correlated with milder residual tumor burden, and ROC curve analysis also supported the predictive value of CXCL16 in NAC response prospect. Furthermore, we also noticed an obvious association between CXCL16 sufficiency and CD4+/CD8+ TILs recruitment. These findings suggested that CXCL16 functions as a favorable factor for tumor response as well as antitumor immune in TNBC cases.

Increasing studies have reported associations between MMPs family members and tumor immunotherapy. The MMPs are zinc-dependent endopeptidases (23), which mediate degradation of various molecules for cell adhesion and modulation of cellular and extracellular matrix interactions (24). Previous studies reported that MMPs were significantly associated with tumor microenvironment and immune cells, thereby targeting MMPs could theoretically solve the problem of immune suppression (25). The MMPs were shown to process CCL/CXCL chemokines and their receptors to modulate antitumor immune responses (26), and they could also contribute to tumor immune escape by inducing the expansion of myeloid-derived suppressor cells (MDSCs) (27). Clinical trials have pharmacologically targeted MMPs, and an MMP inhibitor was also evaluated in phase III trials for patients with breast cancer (28). Our research provides further understanding of MMPs involved in immunotherapy. We observed significant upregulation of MMP7 expression in poor remission cases, which caused an increase of the RCB score. The ROC curve also showed the predictive value of MMP7 in NAC response prospect. Furthermore, over expression of MMP7 suppressed the recruitment of CD4+/CD8+ TILs. These findings suggested that MMP7 functioned as an adverse factor for tumor response as well as antitumor immune in TNBC cases. We also observed up-regulation of CXCL16 in vitro due to the inhibition of MMP7, which implied the modulating effect of MMP7 on CXCL16 expression.

Previous studies have also shown that over-expression of YAP/TAZ is widely involved in migration and invasion of breast cancer cells (29). Knockdown of YAP/TAZ could reduce the above migration and invasion (30). Depletion of YAP/TAZ was outstanding in basal and epithelial cells which promoted luminal differentiation, acting as a luminal to basal lineage switch (31). Prior studies also reported the similar transforming potential of YAP. Overexpression of YAP induced inhibition of apoptosis and anchorage-independent growth, which contributed to the tumorigenic transformation (32). Inhibition of YAP expression suppressed tumor development and metastasis in a breast cancer mouse model (33). At the preclinical research level, YAP/TAZ played an important role in cancer-associated features such as tumor cell migration and invasion. At the clinical study level, YAP/TAZ correlated with tumor metastases and drug resistance, which obviously affected patients` survival. Based on our work in this study, YAP1 was shown to have a close relationship with NAC response as well as anti-tumor immune in TNBC cases. Firstly, up-regulation of YAP1 expression was outstanding in poor response cases, whereas YAP1 insufficient cases performed to a remarkable advantage in RCB. The ROC curve also showed the predictive value of YAP1 in NAC response prospect. Secondly, over expression of YAP1 obviously suppressed recruitment of CD4+/CD8+ TILs according to TCGA data, and CD4+/CD8+TILs counts closely coincided with the YAP1 intensity according to our microscopic observations in this study. Considering the association of MMP7/CXCL16 with TILs recruitment and NAC response, we suspected the potential existence of the YAP1-MMP7-CXCL16 axis. Surprisingly, the relationship between MMP7/CXCL16 and HIPPO-YAP1 relevant kinases expression (such as MST1 and LATS2) was significant. Results of GSEA also supported the regulation effect of HIPPO-YAP1 pathway in MMP7 and CXCL16 expression. Furthermore, we observed that YAP1 inhibition resulted in up-regulation of CXCL16 as well as downregulation of MMP7 in vitro. Thus, we believed that YAP1 over expression caused activation of YAP1-MMP7-CXCL16 axis, which suppressed CD4+/CD8+ TILs recruitment and indirectly affected NAC response of TNBC patients. 
Although YAP1 has been improved to be a crucial factor in the anti-tumor immune suppression (34), the concrete mechanism is still unclear $(35,36)$. Based on the TCGA data, we noticed that enrichment of YAP1-related signal pathway was correlated with MMP7/CXCL16 intensity. We further proved the relationship between YAP1 and MMP7/CXCL16, while the latter played important role in the recruitment of anti-tumor $\mathrm{T}$ cells. Considering the above findings, we supposed that activation of YAP1/ MMP7/CXCL16 axis suppressed TIL recruitment, and over-expression of YAP1 was at the core of TIL activity inhibition. Given the results of our own study and the TCGA database, we firstly established the hypothesis of YAP1/MMP7/CXCL16 axis related anti-tumor immune suppression. This may be a fresh perspective to explore the immune-inhibitory effect of YAP1.Despite the limitations of current conclusion, we still try to explore the relationship between YAP1 and TIL recruitment, thereby improving the therapeutic efficacy of TNBC.

Due to the sample size and follow-up span, there were ineluctable shortcomings in this study. Considering the limitations of this study being a single-center retrospective study with fewer cases, we still require multi-center prospective trials with lager sample sizes to identify the present findings. Continuous improvement of statistical methods is necessary to the advancement of clinical studies. We would also carry out further analysis and update our thesis. We would also adhere to extend the follow-up, thereby obtaining more reliable conclusion.

In conclusion, up-regulation of YAP1/MMP7 and downregulation of CXCL16 were both significant in TNBC cases with poor NAC response. Inhibition of YAP1 induced downregulation of MMP7 and up-regulation of CXCL16, whereas inhibition of MMP7 also induced up-regulation of CXCL16. The YAP1-related pathway was also enriched by MMP7/ CXCL16. Activation of the YAP1/MMP7/CXCL16 axis obviously affected RCB of TNBC cases. The ROC curve also demonstrated the predictive value of the YAP1/MMP7/ CXCL16 axis and TILs density in NAC response prospect. The density of TILs, meanwhile, demonstrated a strong link with YAP1/MMP7/CXCL16 axis. Overexpression of YAP1/ MMP7 significantly suppressed recruitment of CD4+/CD8+ TILs, while CXCL16 over expression had a beneficial impact on anti-tumor immune response.

\section{Acknowledgments}

Funding: None.

\section{Footnote}

Reporting Checklist: The authors have completed the MDAR reporting checklist. Available at https://dx.doi. org/10.21037/gs-21-612

Data Sharing Statement: Available at https://dx.doi. org/10.21037/gs-21-612

Conflicts of Interest: All authors have completed the ICMJE uniform disclosure form (available at https://dx.doi. org/10.21037/gs-21-612). The authors have no conflicts of interest to declare.

Etbical Statement: The authors are accountable for all aspects of the work in ensuring that questions related to the accuracy or integrity of any part of the work are appropriately investigated and resolved. The study was conducted in accordance with the Declaration of Helsinki (as revised in 2013) and was approved by the Clinic Institutional Review Board of Xiangya Hospital. All participants gave our group permission to use their tissue specimens by signing informed consent.

Open Access Statement: This is an Open Access article distributed in accordance with the Creative Commons Attribution-NonCommercial-NoDerivs 4.0 International License (CC BY-NC-ND 4.0), which permits the noncommercial replication and distribution of the article with the strict proviso that no changes or edits are made and the original work is properly cited (including links to both the formal publication through the relevant DOI and the license). See: https://creativecommons.org/licenses/by-nc-nd/4.0/.

\section{References}

1. Zhang G, Qian X, Ren C, et al. GSTM1 polymorphism in Oncotype DX assay is a potential predictive factor for taxane-based neoadjuvant chemotherapy in estrogen receptor-positive Chinese breast cancer patients. Transl Cancer Res 2019;8:655-63.

2. Zheng S, Bai JQ, Li J, et al. The pathologic characteristics of breast cancer in China and its shift during 1999-2008: a national-wide multicenter cross-sectional image over 10 years. Int J Cancer 2012;131:2622-31.

3. Mahamodhossen YA, Liu W, Rong-Rong Z. Triplenegative breast cancer: new perspectives for novel therapies. Med Oncol 2013;30:653. 
4. Andre F, Dieci MV, Dubsky P, et al. Molecular pathways: involvement of immune pathways in the therapeutic response and outcome in breast cancer. Clin Cancer Res 2013;19:28-33.

5. Apetoh L, Ghiringhelli F, Tesniere A, et al. Toll-like receptor 4-dependent contribution of the immune system to anticancer chemotherapy and radiotherapy. Nat Med 2007;13:1050-9.

6. Demaria S, Volm MD, Shapiro RL, et al. Development of tumor-infiltrating lymphocytes in breast cancer after neoadjuvant paclitaxel chemotherapy. Clin Cancer Res 2001;7:3025-30.

7. Galon J, Costes A, Sanchez-Cabo F, et al. Type, density, and location of immune cells within human colorectal tumors predict clinical outcome. Science 2006;313:1960-4.

8. Salgado R, Denkert C, Demaria S, et al. The evaluation of tumor-infiltrating lymphocytes (TILs) in breast cancer: recommendations by an International TILs Working Group 2014. Ann Oncol 2015;26:259-71.

9. Gao X, Ma T, Bai S, et al. A CT-based radiomics signature for evaluating tumor infiltrating Treg cells and outcome prediction of gastric cancer. Ann Transl Med 2020;8:469.

10. Zitvogel L, Kepp O, Kroemer G. Immune parameters affecting the efficacy of chemotherapeutic regimens. Nat Rev Clin Oncol 2011;8:151-60.

11. Greten TF, Ormandy LA, Fikuart A, et al. Low-dose cyclophosphamide treatment impairs regulatory $\mathrm{T}$ cells and unmasks AFP-specific CD4+ T-cell responses in patients with advanced HCC. J Immunother 2010;33:211-8.

12. Loi S, Sirtaine N, Piette F, et al. Prognostic and predictive value of tumor-infiltrating lymphocytes in a phase III randomized adjuvant breast cancer trial in node-positive breast cancer comparing the addition of docetaxel to doxorubicin with doxorubicin-based chemotherapy: BIG 02-98. J Clin Oncol 2013;31:860-7.

13. Ibrahim EM, Al-Foheidi ME, Al-Mansour MM, et al. The prognostic value of tumor-infiltrating lymphocytes in triple-negative breast cancer: a meta-analysis. Breast Cancer Res Treat 2014;148:467-76.

14. Adams S, Gray RJ, Demaria S, et al. Prognostic value of tumor-infiltrating lymphocytes in triple-negative breast cancers from two phase III randomized adjuvant breast cancer trials: ECOG 2197 and ECOG 1199. J Clin Oncol 2014;32:2959-66.

15. Quintana E, Shackleton M, Sabel MS, et al. Efficient tumour formation by single human melanoma cells. Nature 2008;456:593-8.

16. Wilbanks A, Zondlo SC, Murphy K, et al. Expression cloning of the STRL33/BONZO/TYMSTR ligand reveals elements of CC, CXC, and CX3C chemokines. J Immunol 2001;166:5145-54.

17. Matloubian M, David A, Engel S, et al. A transmembrane CXC chemokine is a ligand for HIV-coreceptor Bonzo. Nat Immunol 2000;1:298-304.

18. Liao F, Alkhatib G, Peden KW, et al. STRL33, A novel chemokine receptor-like protein, functions as a fusion cofactor for both macrophage-tropic and $\mathrm{T}$ cell line-tropic HIV-1. J Exp Med 1997;185:2015-23.

19. Hase K, Murakami T, Takatsu H, et al. The membranebound chemokine CXCL16 expressed on follicle-associated epithelium and $M$ cells mediates lympho-epithelial interaction in GALT. J Immunol 2006;176:43-51.

20. Shimaoka T, Nakayama T, Fukumoto N, et al. Cell surface-anchored SR-PSOX/CXC chemokine ligand 16 mediates firm adhesion of CXC chemokine receptor 6-expressing cells. J Leukoc Biol 2004;75:267-74.

21. Gao JQ, Sugita T, Kanagawa N, et al. Anti-tumor responses induced by chemokine CCL19 transfected into an ovarian carcinoma model via fiber-mutant adenovirus vector. Biol Pharm Bull 2005;28:1066-70.

22. Tada T, Ohzeki S, Utsumi K, et al. Transforming growth factor-beta-induced inhibition of $\mathrm{T}$ cell function. Susceptibility difference in $T$ cells of various phenotypes and functions and its relevance to immunosuppression in the tumor-bearing state. J Immunol 1991;146:1077-82.

23. Verma RP, Hansch C. Matrix metalloproteinases (MMPs): chemical-biological functions and (Q)SARs. Bioorg Med Chem 2007;15:2223-68.

24. Kessenbrock K, Plaks V, Werb Z. Matrix metalloproteinases: regulators of the tumor microenvironment. Cell 2010;141:52-67.

25. Li M, Xing S, Zhang H, et al. A matrix metalloproteinase inhibitor enhances anti-cytotoxic $\mathrm{T}$ lymphocyte antigen-4 antibody immunotherapy in breast cancer by reprogramming the tumor microenvironment. Oncol Rep 2016;35:1329-39.

26. McQuibban GA, Gong JH, Wong JP, et al. Matrix metalloproteinase processing of monocyte chemoattractant proteins generates CC chemokine receptor antagonists with anti-inflammatory properties in vivo. Blood 2002;100:1160-7.

27. Shao L, Zhang B, Wang L, et al. MMP-9-cleaved osteopontin isoform mediates tumor immune escape by inducing expansion of myeloid-derived suppressor cells. Biochem Biophys Res Commun 2017;493:1478-84.

28. Brown PD. Ongoing trials with matrix metalloproteinase 
inhibitors. Expert Opin Investig Drugs 2000;9:2167-77.

29. Mi W, Lin Q, Childress C, et al. Geranylgeranylation signals to the Hippo pathway for breast cancer cell proliferation and migration. Oncogene 2015;34:3095-106.

30. Chan SW, Lim CJ, Guo K, et al. A role for TAZ in migration, invasion, and tumorigenesis of breast cancer cells. Cancer Res 2008;68:2592-8.

31. Skibinski A, Breindel JL, Prat A, et al. The Hippo transducer TAZ interacts with the SWI/SNF complex to regulate breast epithelial lineage commitment. Cell Rep 2014;6:1059-72.

32. Overholtzer M, Zhang J, Smolen GA, et al. Transforming properties of YAP, a candidate oncogene on the chromosome 11q22 amplicon. Proc Natl Acad Sci U S A 2006;103:12405-10.

33. Chen Q, Zhang N, Gray RS, et al. A temporal requirement for Hippo signaling in mammary gland differentiation, growth, and tumorigenesis. Genes Dev 2014;28:432-7.

34. Lebid A, Chung L, Pardoll DM, et al. YAP Attenuates CD8 T Cell-Mediated Anti-tumor Response. Front Immunol. 2020;11:580.

35. Hojo S, Koizumi K, Tsuneyama K, et al. High-level expression of chemokine CXCL16 by tumor cells correlates with a good prognosis and increased tumorinfiltrating lymphocytes in colorectal cancer. Cancer Res 2007;67:4725-31.

36. Pinard C, Debled M, Ben Rejeb H, et al. Residual cancer burden index and tumor-infiltrating lymphocyte subtypes in triple-negative breast cancer after neoadjuvant chemotherapy. Breast Cancer Res Treat 2020;179:11-23.

(English Language Editor: J. Jones)
Cite this article as: Yuan JQ, Zhang KJ, Wang SM, Guo L. YAP1/MMP7/CXCL16 axis affects efficacy of neoadjuvant chemotherapy via tumor environment immunosuppression in triple-negative breast cancer. Gland Surg 2021;10(9):27992814. doi: $10.21037 / g s-21-612$ 